\title{
IP Datacasting and Channel Error Handling with DVB-H
}

\author{
Jason Yao \\ Weifeng Huang \\ Ming-Syan Chen \\ Graduate Institute of Communication Graduate Institute of Communication Graduate Institute of Communication \\ Engineering \\ Engineering \\ Engineering \\ National Taiwan University \\ jasonyao@cc.ee.ntu.edu.tw \\ National Taiwan University \\ weifeng@arbor.ee.edu.tw \\ National Taiwan University \\ mschen@cc.ee.ntu.edu.tw
}

\begin{abstract}
DVB-H is a terrestrial digital TV standard which consumes less power and allow user to move freely when receiving signals. Its deployment also signifies the convergence between broadcast network and data networks as both video signals and other data programs are transmitted in a shared media. In this paper we discuss the channel error problems under different scenarios of this convergence. Partial solutions, such as enhanced Forward Error Correction were provided by the standard but other aspects still need further exploration. We present some early results and discuss possible directions in the future.
\end{abstract}

\section{Keywords}

Digital Video Broadcasting, DVB-H, Forward Error Correction, IP datacasting, MANET

\section{INTRODUCTION}

DVB-T [1], the European standard for terrestrial digital video broadcast system is opening up a huge capacity for transmission of digital content. Another standard, DVB-H [2] supports mobility. Its datacasting capability can also be used to deliver not only digital multimedia but also digital services. Consequently massive audiences can be reached anywhere, anytime if suitable devices are available. A new platform for broadband services and universal access is emerging as more devices are equipped with DVB-H functions at lower costs.

Figure 1 shows a high-level diagram of datacasting [3]. Consumers can benefit from datacasting because they have more choices on access to multimedia content, in addition to web browsing with PCs or with cellular phones. Datacast may also stimulate content providers to offer more diverse programs as it becomes economically feasible to distribute and charge for media products targeted for smaller audiences. For example, governments and public institutions may use datacasting to provide educational programs in cost efficient ways for citizens.

While datacasting with DVB-H can support the distribution of new programs, the wireless transmission environment can be plagued by channel errors and the broadcast nature of the service makes it difficult to retransmit the lost pieces to each and every receivers. For audio/video programs, lost data means degradation in subjective quality and can be tolerated if the duration is short. Other types of data, software downloads for example, can be totally useless even if just one single bit is wrong. Thus we need to carefully examine the channel error handling functions of DVB-H.

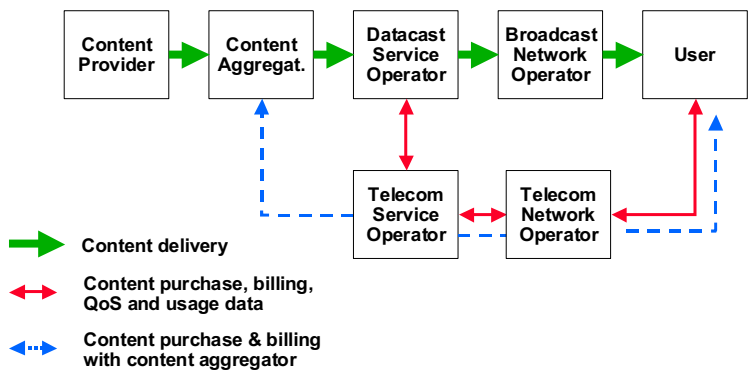

Figure 1 Generic Model in Datacasting

The rest of this paper is organized as follows. Background information of DVB-H is briefly introduced in Section 2. The FEC and return channel issues of the system are discussed in Section 3. Section 4 presents some experimental results and discusses possible scenarios. Section 5 concludes the paper and gives future directions of our research.

\section{DVB-H}

Digital Video Broadcasting (DVB) [1] systems have been widely deployed in recent years, providing a standard way of carrying multimedia services over digital terrestrial broadcasting networks in many countries. DVB-H (Handheld) [2], fully compatible with DVB-T, gives additional features to support portable reception, including power saving, mobility, general robustness, noise tolerance and seamless handover, which have been achieved by combining elements in the physical and link layers as well as service information.

In the link layer, DVB-H make use of Time-Slicing, reducing the average power consumption of terminals and enabling smooth and seamless frequency handover. It also offers the option of extra Forward Error Correction to improve performance in mobile channels degraded by 
impulse interference and Doppler Effect. In physical layer, DVB-H added 4K-mode for trading off mobility and SFN cell size. It should be mentioned that the payload of DVB$\mathrm{H}$ are IP-datagrams or other network layer datagrams encapsulated into MPE-sections.

\section{CHANNEL ERROR CONSIDERATIONS}

When the wireless channel is not reliable, the system can use redundancies to increase to probability of correct decoding of the source data. However, higher redundancies imply lower bandwidth available for source data transmission, thus it is necessary to select the best protection scheme according to the channel conditions. In order to respond to the time-varying nature of channels, the system needs a feedback channel to provide channel information to the broadcast side.

\subsection{Forward Error Correction}

DVB uses the MPEG-2 format for its video content and in MPEG source coding, redundancy and irrelevance are mostly removed from the coded bits, which also become more sensitive to disturbances during transmission. A bit error occurs when the noise level exceeds a threshold at the receiver. Therefore, forward error correction (FEC) is designed to prevent such faulty outcome by adding appropriate redundancy bits.

Error control codes can be divided to two classes, block codes and convolutional codes. In the case of block codes, the input data stream is divided into blocks of a fixed length $\mathrm{m}$, where $\mathrm{m}$ is the number of symbols. Such symbols can either be comprised of one bit or several bits. The error correction must not only find the erroneous symbol, but also determine the original value of the symbol. In block codes, the calculated redundancy is appended to the actual $\mathrm{m}$ information positions in the $\mathrm{k}$ correction positions. So that finally a block with a length of $n=m+k$ is transmitted. The code rate indicates the ratio between the information $\mathrm{m}$ and the transmission symbols $\mathrm{n}$. One example of block codes is Reed-Solomon codes.

The convolutional codes differ from the block codes in that binary shift registers are used, without dividing the input stream into segments. Convolutional codes are designed to correct individual bit errors and the code rate is defined as the ratio of the number of input bits to the number of output bits generated continuously.

The standard design of FEC in DVB includes the concatenation of a Reed-Solomon codes unit and a convolutional codes unit, plus a interleaver, as shown in Figure 2.

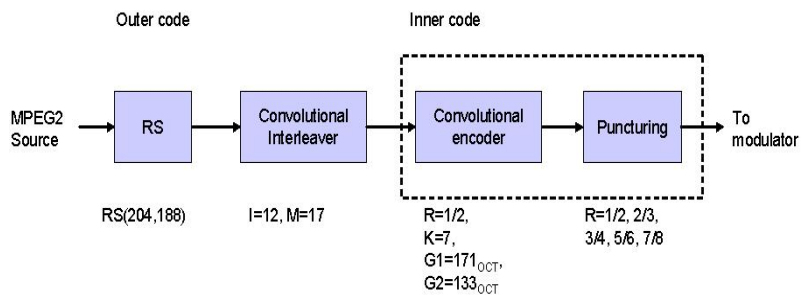

Figure 2 Forward Error Correction Model in DVB

\subsection{Return Channel}

A way to add interactivity to the communication system defined by DVB is to make use of the two-way capabilities of GSM/GPRS which provides a wireless, bi-directional data channel connecting users to service providers. A GSM mobile station (MS) forms the interactive interface unit with access to GSM networks from user terminals.

By utilizing return channels, a feedback representing current network condition observed by receiving end can be sent back the local service provider, as shown in Figure 3 .

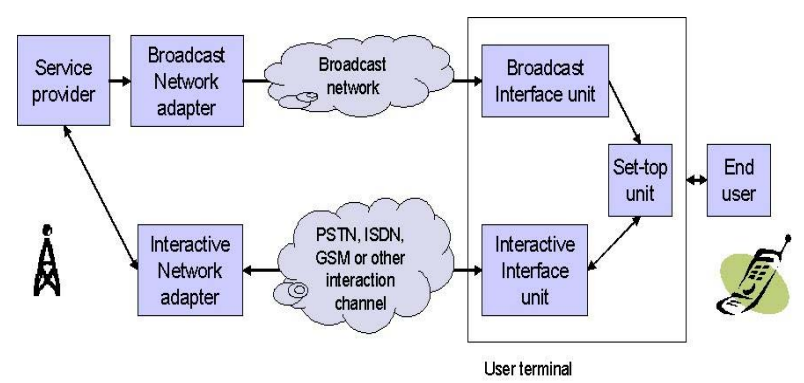

Figure 3 Broadcasting and Return channel

Assuming that the end user device is capable of communicating with the local broadcast service provider and reporting an accurate estimation of the channel error condition, it remains a difficult task to aggregate all the information provided by multiple receivers that may experience very different channel conditions. We will discuss these issues in the next section.

\section{DISCUSSIONS}

In a separate detailed study [4], we have simulated the additional FEC functions available in DVB-H that offer up to $64 \mathrm{RS}$ redundancy bits per frame. A typical result is shown in Figure 4. 


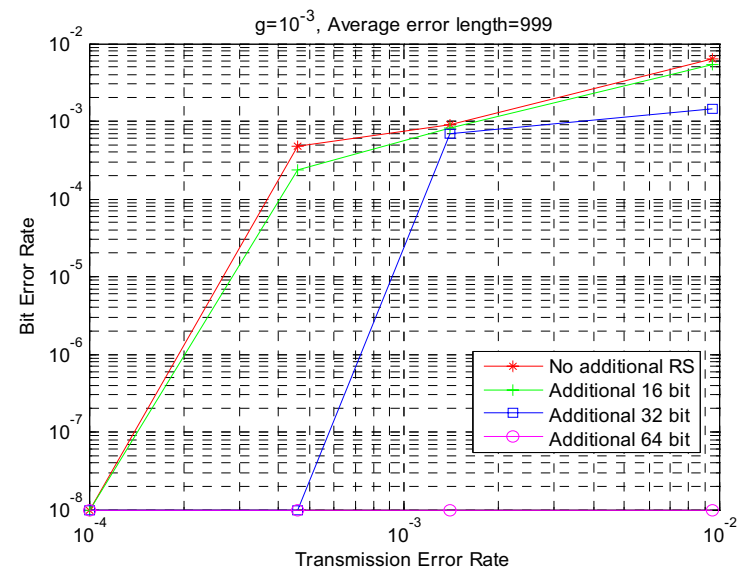

Figure 4 Results of Additional FEC in DVB-H

As expected, the additional FEC improves the error rate with higher redundancies. However, it is only effective in a certain range. Within this range, we have designed an Adaptive Error control Scheme [4] which offers different algorithms for two content types: (1) Multimedia, (2) Program Data. The former provides general error control since human perception can tolerate some errors in audio and video. The latter adopts more stringent bit error control due to high error-sensitivity of program data. The proposed adaptive method achieves better throughput than ones with fixed FEC methods under time-varying channel conditions.

That said, we have to recognize two factors that are beyond repair by the additional FEC scheme. First, it does not work when the error rate is too high. This can happen when the receiver runs into a blind spot not covered by the broadcast station. Second, the channel feedback may not be timely and accurate. There is always a delay before the broadcaster is aware of deterioration in channel conditions. Beside, it may not be efficient to use excessive FEC just for a few users with poor reception. Under these conditions, data loss would surely occur for some receivers and so far there is no standard remedy in standard DVB-H.

Two categories of solutions can be envisioned.
1. Client-Server solution. The receiver may obtain the lost data via their GSM/GPRS network. Since they have the connection for channel condition feedback, they may simultaneously request for the lost data. This solution is straightforward although the server may get inundated with requests when suddenly the broadcast channel gets too noisy.

2. Peer-to-peer solution. In case that receivers can be organized to an ad-hoc network (Mobile Ad hoc Network, MANET), they can ask for the missing pieces from their peers without connecting to a server. However, they may still need to retrieve some data from servers if they cannot find a local copy.

\section{CONCLUSION}

In this paper, we explore the scenarios of IP datacasting with DVB-H. The standard provides a good framework for both audio/video and data services but it is not enough to achieve lossless data transfer when severe channel error occurs. We examined the FEC enhancement scheme which alleviates the problem under certain conditions and suggested two solutions that may be incorporated for ultimate data recovery. In the future, we would look into the scenario of peer-to-peer solution, which should be more scalable. This method would be compared with the clientserver solution, taking into account of cost, which includes speed of recovery, power consumption, additional hardware and communications overhead.

\section{REFERENCES}

[1] ETSI EN 300744 "Digital Video Broadcasting (DVB); Framing structure, channel coding and modulation for ditigal terrestrial television, November 2004

[2] ETSI DVB Document A081, Transmission System for Handheld Terminals (DVB H), June 2004

[3] IPDC Article for BCE 3 "http://www.ipdcforum.org/resources/resources.html

[4] Master's Thesis by Weifeng Huang, National Taiwan University, 2005 